\title{
Mouse Primary Visual Cortex Is Used to Detect Both Orientation and Contrast Changes
}

\author{
Lindsey L. Glickfeld, ${ }^{\star}$ Mark H. Histed, ${ }^{\star}$ and John H. R. Maunsell \\ Department of Neurobiology, Harvard Medical School, Boston, Massachusetts 02115
}

In mammals, the lateral geniculate nucleus (LGN) and the superior colliculus (SC) are the major targets of visual inputs from the retina. The LGN projects mainly to primary visual cortex (V1) while the SC targets the thalamus and brainstem, providing two potential pathways for processing visual inputs. Indeed, cortical lesion experiments in rodents have yielded mixed results, leading to the hypothesis that performance of simple visual behaviors may involve computations performed entirely by this subcortical pathway through the SC. However, these previous experiments have been limited by both their assays of behavioral performance and their use of lesions to change cortical activity. To determine the contribution of $\mathrm{V} 1$ to these tasks, we trained mice to perform threshold detection tasks in which they reported changes in either the contrast or orientation of visual stimuli. We then reversibly inhibited V1 by optogenetically activating parvalbumin-expressing inhibitory neurons with channelrhodopsin-2. We found that suppressing activity in V1 substantially impaired performance in visual detection tasks. The behavioral deficit depended on the retinotopic position of the visual stimulus, confirming that the effect was due to the specific suppression of the visually driven V1 neurons. Behavioral effects were seen with only moderate changes in neuronal activity, as inactivation that raised neuronal contrast thresholds by a median of only $14 \%$ was associated with a doubling of behavioral contrast detection threshold. Thus, detection of changes in either orientation or contrast is dependent on, and highly sensitive to, the activity of neurons in V1.

Key words: contrast; inhibition; mouse visual cortex; optogenetics; orientation; psychophysics

\section{Introduction}

Certain brain regions have been shown to control specific behaviors. For instance, lesions of the human Broca's and Wernicke's areas have precise and nonoverlapping effects on speech production (Damasio and Geschwind, 1984). However, for many types of behavior, it has been difficult to assign specific roles to particular brain regions. Instead, lesions of one region are often compensated for by a different region, as in the case of eye movements, which can be controlled by either of two different areas (Schiller et al., 1980). Beyond this sort of redundancy, adaptation and learning can also allow recovery of function after lesions (e.g., in V1; Dean, 1978). Effects like these probably contributed to Lashley's famous conclusions that any cortical area can take over function from any other (Lashley, 1931a). Although we now know this is not generally true, redundancy, adaptation, and learning still hinder our ability to determine the contribution of specific brain areas to behavior.

\footnotetext{
Received Aug. 20, 2013; revised 0ct. 30, 2013; accepted Nov. 2, 2013.

Author contributions: L.L.G., M.H.H., and J.H.R.M. designed research; L.L.G. and M.H.H. performed research; L.L.G. and M.H.H. analyzed data; L.L.G., M.H.H., and J.H.R.M. wrote the paper.

This project was supported by funding from the US National Institutes of Health (R01 EY018742) and a Fellowship from the Helen Hay Whitney Foundation (L.L.G.). We thank A. Handler, A. McKinney, and S. Sleboda for technical support, V. Berezovskii and N. Comfort for assistance with histology, C. Reid for helpful discussions, and M. Andermann and G. Horwitz for useful comments on this manuscript.

*L.L.G. and M.H.H. contributed equally to this work.

Correspondence should be addressed to Lindsey L. Glickfeld at her current address: Department of Neurobiology, Duke University, Durham, NC 27710. E-mail: glickfeld@neuro.duke.edu.

DOI:10.1523/JNEUROSCI.3560-13.2013

Copyright $\odot 2013$ the authors $\quad 0270-6474 / 13 / 3319416-07 \$ 15.00 / 0$
}

Thus, reversible silencing is a powerful approach for understanding an area's role in behavior. Optogenetic manipulations can achieve this by rapidly modulating the activity of specific brain regions and cell types. In particular, optogenetic activation of parvalbumin-expressing $(\mathrm{PV}+)$ inhibitory interneurons can reversibly silence neuronal activity (Cardin et al., 2009; Sohal et al., 2009; O'Connor et al., 2013). The majority of PV + interneurons are basket cells that target the soma and proximal dendrites of cortical pyramidal cells (Kawaguchi and Kubota, 1997) and generate a strong and fast inhibitory conductance (Gabernet et al., 2005; Packer and Yuste, 2011) that decreases local excitability (Atallah et al., 2012; Lee et al., 2012; Wilson et al., 2012). Thus by infecting and activating PV + interneurons with channelrhodopsin-2 (ChR2), we can transiently suppress neuronal activity in primary visual cortex (V1).

Here, we use this approach to determine whether mouse V1 is involved in a visual detection task. When investigated with ablation, the role of V1 in rodents has been historically unclear. Some studies have found that V1 is required for detection and discrimination of visual features (Lashley, 1931b; Petruno et al., 2013), whereas others argue that V1 is required for discrimination but not detection (Schneider, 1969), and yet others identified only subtle changes in visual acuity (Dean, 1981). The most consistent finding across studies is the requirement of $\mathrm{V} 1$ for processing higher spatial frequencies (Lashley, 1939; Dean, 1978; Prusky and Douglas, 2004). On the one hand, the finding that V1 may not be critical for visual perception is unsurprising given the comparative importance of subcortical visual pathways in rodents (Hof- 
A
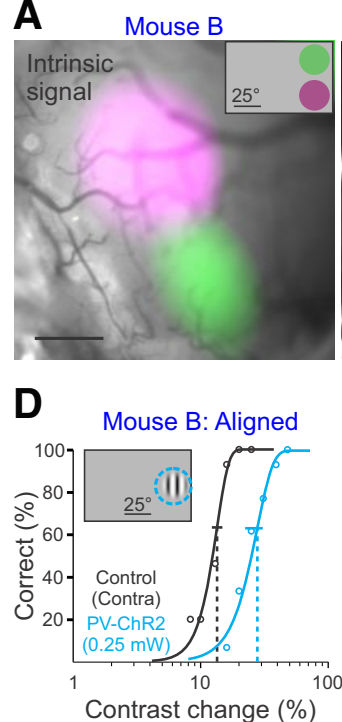

Figure 1. Activation of PV + interneurons increases contrast change detection threshold. $\boldsymbol{A}$, Left, Intrinsic autofluorescence signal in response to visual stimuli presented in two positions, overlaid on a raw fluorescence image of the cranial window. Inset, Relative position and extent of the two visual stimuli (green, $35^{\circ}$ azimuth, $20^{\circ}$ elevation; purple, $35^{\circ}$ azimuth, $-20^{\circ}$ elevation). Right, ChR2-mCherry fluorescence from viral infection: the expression spans portions of the green and purple regions confirming the visual field represented by the ChR2 injection. Scale bar, $500 \mu \mathrm{m}$. A, Anterior; P, posterior; L, lateral; M, medial. B, ChR2$\mathrm{mCherry}$ fluorescence in a coronal section. WM, White matter. Scale bar, $100 \mu \mathrm{m}$. C, Schematic of trial progression. At the end of the intertrial interval, the trial begins when the mouse depresses the lever. A visual stimulus may appear from 400 to $4000 \mathrm{~ms}$ from the onset of the trial; the mouse must release the lever $100-550 \mathrm{~ms}$ after the stimulus appears to receive a reward. On randomly interleaved trials, ChR2-expressing PV + interneurons are illuminated with $455 \mathrm{~nm}$ light for the duration of the trial. $\boldsymbol{D}$, Representative behavioral performance, single session. Stimulus presented in contralateral field of view (relative to the ChR2 injection site) during control trials (black) and during activation of PV + interneurons (blue) as stimulus contrast is varied (same mouse as in $A$ ). Data are fit with a Weibull function to determine detection thresholds (dotted vertical lines) and $95 \%$ confidence intervals (solid horizontal lines). Inset, Schematic of retinotopic position of visual stimulus and ChR2 suppression (dotted blue circle). E, Same as on left for presentation of a stimulus in the ipsilateral field of view. $F$, Summary of effect of activation of PV + interneurons at a single power $(0.25 \mathrm{~mW}$; not corrected for power density) on behavioral performance at three visual stimulus positions for three mice ( $\mathrm{N}$, numbers of behavioral sessions per mouse). Dotted line represents no change in detection threshold (threshold ratio $=1$ ). All error bars indicate \pm SEM.

bauer and Drager, 1985). On the other hand, the use of permanent lesions in those experiments, and the relearning and adaptation that follows, complicates their interpretation (Dean, 1978, 1981).

To examine the behavioral role of mouse $\mathrm{V} 1$, we reversibly suppressed neuronal activity during a perceptually demanding behavioral task. Our approach reveals that V1 is used for the detection of both increments and decrements in contrast as well as changes in orientation. Thus, despite the prominent retinocollicular projection, even simple visual detection behavior relies on computations in $\mathrm{V} 1$.

\section{Materials and Methods}

Animals. All animal procedures were conducted in accordance with the ethical guidelines of the NIH and approved by the Institutional Animal Care and Use Committee at Harvard Medical School. Three PV-Cre mice were used (JAX stock \#008069; The Jackson Laboratory; Hippenmeyer et al., 2005; C57BL/6 background; 2 female, 1 male; singly housed on a reverse light/dark cycle). The animals are designated A, B, and C consistently throughout the report.

Cranial window implant. Mice (4-5 months old) were implanted with a headpost and cranial window to give stable optical access for photostimulation during behavior. Animals were anesthetized with ketamine (40 mg/kg, i.p.), xylazine ( $2 \mathrm{mg} / \mathrm{kg}$, i.p.) and isoflurane $(1.2-2 \%$ in $100 \%$ $\mathrm{O}_{2}$ ), and dexamethasone ( $3.2 \mathrm{mg} / \mathrm{kg}, \mathrm{i} . \mathrm{m}$.) was administered at least $2 \mathrm{~h}$ before surgery. Using aseptic technique, a headpost was secured using C\&B Metabond (Parkell), and a $3 \mathrm{~mm}$ craniotomy was made over the left cerebral hemisphere (center: $2.8 \mathrm{~mm}$ lateral, $0.5 \mathrm{~mm}$ anterior to lambda) to implant a glass window (thickness, $0.8 \mathrm{~mm}$ ).

Intrinsic autofluorescence imaging. To map $\mathrm{V} 1$, we measured changes in the intrinsic autofluorescence signal using epifluorescence imaging (Andermann et al., 2011). Autofluorescence produced by blue excitation $(470 \pm$ $40 \mathrm{~nm}$, Chroma) was collected through a green longpass emission filter (500 nm cutoff) and a $5 \times$ air objective $(0.14$ numerical aperture, $\mathrm{Mi}$ tutoyo) onto a CCD camera (Sensicam, Cooke; $344 \times 260$ pixels spanning $4 \times 3 \mathrm{~mm} ; 2 \mathrm{~Hz}$ acquisition rate) using NIH ImageJ acquisition software. For retinotopic mapping, we presented upward drifting gratings $\left(40^{\circ}\right.$ diameter Gabor patch; $2 \mathrm{~Hz}$ and 0.04 cycles/degree) for $10 \mathrm{~s}$, with $10 \mathrm{~s}$ of mean luminance preceding each trial. The response to a stimulus was computed as the fractional change in fluorescence between the average of all frames from 0 to $10 \mathrm{~s}$ (as baseline) and from 11 to $20 \mathrm{~s}$ (as response) after stimulus onset.

Viral injections and ChR2 stimulation. We targeted the medial monocular region of $\mathrm{V} 1$ (corresponding to $\sim 35^{\circ}$ in azimuth and $0^{\circ}$ in elevation) for ChR2 expression. Dexamethasone was administered at least $2 \mathrm{~h}$ before cover slip removal. Mice were anesthetized (isoflurane, $1-1.5 \%)$, the cranial implant was sterilized with alcohol, and the cover slip was removed. We then used a volume injection system $(100 \mathrm{nl} / \mathrm{min}$, Stoelting $)$ to inject $250 \mathrm{nl}$ of a 10:1 mixture of AAV2/8-DIO-ChR2-mCherry $\left(10^{8}-10^{9}\right.$ viral particles) and sulforhodamine101 (100 $\mu \mathrm{M}$; Invitrogen) to visualize the injection at a depth of $200-300 \mu \mathrm{m}$ below the pial surface. After the injection, a new cranial window was sealed in place.

Several weeks after injection, we localized the area of expression using mCherry fluorescence (Fig. $1 \mathrm{~A})$ and fixed an optical fiber $(400$ $\mu \mathrm{m}$ diameter; Doric Lenses) within $500 \mu \mathrm{m}$ of the cranial window $(\sim 1.3$ $\mathrm{mm}$ above the cortex). We delivered light though the fiber from a $455 \mathrm{~nm}$ LED and calibrated the total light intensity at the entrance to the cannula using a photodiode (Newport). To calculate power density at the dural surface, we normalized the total light intensity by the laser spot size (measured with a CCD camera as the full-width at halfmaximum; range, $0.4-0.8 \mathrm{~mm}^{2}$ ). Experiments were conducted at least 6 weeks after injection.

Behavioral task. Animals were water scheduled and trained to detect changes in visual stimuli by manipulating a lever (Histed et al., 2012). We first trained mice to detect contrast increments on a uniform mean luminance background. On the initial days of training, mice were rewarded for holding the lever for at least $300 \mathrm{~ms}$ (required hold time) but no more than $20 \mathrm{~s}$ (maximum hold time). At the end of the required hold time, a $100 \%$ contrast, full-field grating appeared on the monitor and remained until the mouse released the lever. Typically, within 2 weeks of training, the mice began releasing the lever as soon as the visual stimulus appeared. Once the animals began responding to the visual stimulus, we added a random delay and reduced the maximum hold time. Over the course of the next few months, the task was made harder by increasing the random delay, decreasing the maximum hold time, shortening the stimulus duration, adding additional lower contrast stimuli and reducing the maximum contrast, and shrinking and moving the stimuli to more eccentric positions. Delays after errors were also incorporated to discourage lapses and false alarms. Animals were used for experiments once measured thresholds were stable across multiple days of training. 
The same animals, once trained to detect contrast increments, were subsequently retrained to perform orientation change detection task. A $100 \%$ contrast visual stimulus was continuously displayed; after a random delay, the stimulus underwent a large change in orientation (45-90 degrees) until the mouse released the lever. Animals typically responded to the orientation changes on the first session within the first 10 trials, after which we made the task harder by adding smaller changes in orientation and reducing the maximum change. One mouse was subsequently trained to respond to contrast increments and then decrements of a grating of the same size, location, and spatial frequency, which was continuously displayed. A similar approach of beginning with easy detection trials and then adding harder trials over the course of a few weeks was used for this task as well.

In the final form of the behavioral task, visual stimuli were static Gabor patches $25^{\circ}$ in diameter, positioned at an eccentricity of $35^{\circ}$ in azimuth and $0^{\circ}$ in elevation and presented for $100 \mathrm{~ms}$ (pixel rise time: $2.5 \mathrm{~ms}$ to half-max). The acute viewing angle on the LCD monitor reduced the effective contrast by a factor of $\sim 0.12$ (i.e., $50 \%$ nominal contrast was displayed at $44 \%$ contrast); we did not correct for this small effect as our results depend only on comparing performance between control and PV-ChR2 stimulation conditions at the same stimulus location. Each trial was initiated when the intertrial interval had elapsed and the mouse had depressed the lever (Fig. 1B); during the trial, the stimulus change occurred with a random delay (geometric distribution) after the first $400 \mathrm{~ms}$ of each trial. The relevant stimulus parameter (contrast, orientation, or ChR2 stimulation) was randomly selected from a fixed set of values for each trial to construct psychometric functions. All stimulus onset times were corrected for the monitor's $25 \mathrm{~ms}$ processing latency. All responses occurring earlier than $75 \mathrm{~ms}$ after the visual stimulus change were considered false alarms. All responses occurring $>525 \mathrm{~ms}$ after the change were considered misses. Behavioral control was done with MWorks (http://mworks-project.org) and custom software in Matlab (MathWorks) and Python (http://enthought. com).

Electrophysiology. The cranial window was removed for electrophysiology experiments, but the position of the optical fiber was unchanged to approximate ChR2 stimulation conditions in the behavioral experiments. Recordings were made from the ChR2-mCherry-expressing area with a multisite silicon probe electrode (NeuroNexus; 32 -site model 4x8100-400-177; 4 shanks, 8 sites/shank). We isolated single units and multiunits by amplifying site signals, filtering between $750 \mathrm{~Hz}$ and $7.5 \mathrm{kHz}$, and sampling around threshold crossings (Blackrock). During recording, animals were awake and passively viewing visual stimuli. Each stimulus sequence consisted of 12 presentations (duration of each presentation, $200 \mathrm{~ms}$ with $800 \mathrm{~ms}$ mean luminance between stimuli; duration of stimulus sequence, $12 \mathrm{~s}$ ) of 6 randomly interleaved contrasts; light stimulation of ChR2 was delivered on randomly interleaved stimulus sequences and lasted for the entire sequence. Each recording session consisted of the presentation of at least 100 stimulus sequences.

Histology. Mice were perfused with 4\% paraformaldehyde in PBS, after which the brain was removed and submerged in fixative overnight. The brain was subsequently washed with PBS and place in a $30 \%$ sucrose solution until it sank, and sectioned at $50 \mu \mathrm{m}$ on a freezing microtome. The sections were then mounted on a cover slip and imaged using an Olympus VS120 slide scanner at $20 \times$ to visualize mCherry expression.

Data analysis. All data were analyzed in Matlab (MathWorks). To determine detection thresholds, only those trials in which the subject either responded correctly or failed to respond were considered (Histed et al., 2012). However, if the subject made a false alarm response during the response window, the response would be spuriously counted as correct, artificially improving the subject's performance. To correct for this, we used the false alarm rate to estimate the probability that each correct response was a false alarm. We then removed a proportional number of correct trials based on the length of the reaction time window. Since this is a detection task with a reaction time window much shorter than the possible stimulus window, the correction is small (percentage of trials removed; median, $5.8 \%$; range, $0.8-11.7 \%$; $n=115$ sessions). Corrected response data were fit with Weibull cumulative density functions via least squares weighted by the variance of each mean. We fit the lapse rate (upper asymptote), slope, and threshold (63\% point between upper and lower asymptotes). Threshold confidence intervals (CIs) were estimated via nonparametric bootstrap. We used threshold measurements only from sessions in which (1) the false alarm rate was $<50 \%$, (2) there was sufficient data to yield an accurate threshold estimate (ratio of upper and lower limits of $95 \% \mathrm{CI}<2$ ), and (3) the lapse rate $(100 \%$, fit upper asymptote) was $<10 \%$.

The depth of each electrode was estimated by identifying the most superficial electrode on each shank that registered a modulated LFP ( $\geq 3 \times$ increase in peak-to-peak LFP amplitude compared with electrodes above the cortical surface; LFP was filtered from $0.3-500 \mathrm{~Hz}$ ). The uncertainty of this estimate is the distance between electrode sites (100 $\mu \mathrm{m})$. Waveforms were assigned to units using the OfflineSorter package (Plexon) and designated as single units if the first few principal components and interspike interval (ISI) distributions were distinct from other clusters, and all ISIs were $>1.5 \mathrm{~ms}$. Clusters that overlapped with others at any point in the recording session were classified as multiunits. The spontaneous firing rate was determined by summing all spikes occurring in a $100 \mathrm{~ms}$ window preceding the visual stimulus. Many of the visually responsive neurons had stereotyped temporal dynamics in which there was a rapid increase in firing rate followed by a suppression of firing rate (Haider et al., 2013). Activation of PV + interneurons often reduced both the transient increase and later suppression, sometimes resulting in a paradoxical increase in total firing rate. To avoid these dynamics, we calculated the visually evoked firing rate from the initial $100 \mathrm{~ms}$ of the visual response (25-125 ms after the onset of the visual stimulus), which likely corresponds to the behaviorally relevant epoch. Only shanks which had at least a 25\% ChR2-induced suppression of the spontaneous firing rate (mean across units on that shank) were included for analysis; excluded shanks were generally either outside of the site of ChR2 expression or occluded by blood. Only those units with significantly driven visual responses (as determined by a Kruskal-Wallis test, $p<0.001 ; 85$ of 123 units) were included in this analysis. Each unit's contrast response function under control conditions was fit with a Weibull function with upper and lower saturation, slope, and contrast threshold as free parameters; the response function for ChR2 stimulation trials was then refit (height and slope were fixed to control) to determine changes in threshold. The population response was determined by pooling all spikes from all recorded units.

\section{Results}

To suppress neuronal activity in V1, we conditionally expressed the light-sensitive cation channel ChR2 (Nagel et al., 2003) in $\mathrm{PV}+$ inhibitory neurons, using viral injection into $\mathrm{PV}$-cre mice. In this system, the spatial extent of cortical suppression is limited by the spread of the viral infection. Thus, to ensure that the location of the visual stimulus was retinotopically aligned with the visual field representation in the suppressed region of the cortex, we used intrinsic autofluorescence imaging to target the viral injection to the medial portion of the monocular visual field (Fig. $1 A)$. The extent of ChR2 expression was visualized in vivo by the coexpression of mCherry, revealing that the infection was correctly targeted and limited to $\sim 1 \mathrm{~mm}$ from the injection site (range, $0.7-1.5 \mathrm{~mm}$ ). Histological sections revealed mCherry expression in layers I-V (Fig. $1 B$ ); although the majority of expression was in $\mathrm{PV}+$ interneuronal cell types with multipolar dendritic structures, there was some sparse expression in layer 5 pyramidal cells, as described previously for this mouse line (Tanahira et al., 2009; Urban et al., 2012). Below, we show that the overall effect on neural responses is strongly suppressive when we stimulate these PV+ neurons, suggesting the majority of Creexpressing neurons are indeed inhibitory.

We trained mice to perform a contrast detection task while their heads were held fixed to allow consistent stimulus presentation (Fig. 1C). The mouse initiates a trial by pressing a lever. The computer monitor is initially at mean luminance, and after a 
A

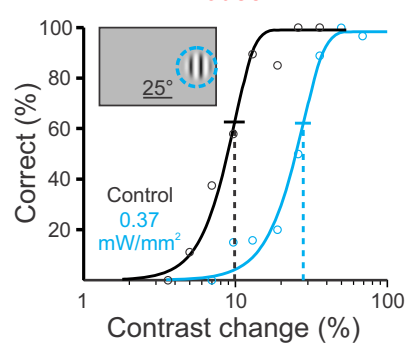

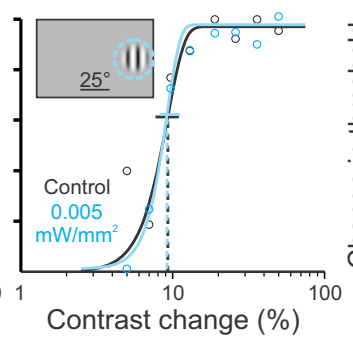

B

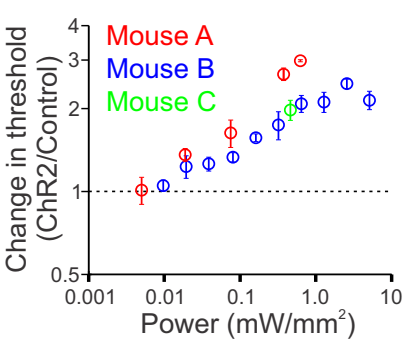

Figure 2. Threshold to detect contrast changes depends on the strength of activation of PV + interneurons. $\boldsymbol{A}$, Representative behavioral performance during control trials (black) and during activation of PV + interneurons (blue) with $0.37 \mathrm{~mW} / \mathrm{mm}^{2}$ (left), showing significant degradation of detection threshold, or $0.005 \mathrm{~mW} / \mathrm{mm}^{2}$ (right), showing little effect. $\boldsymbol{B}$, Summary of effect of activation of PV + interneurons at a range of power densities (light power normalized for area of light spot) for three mice.

as light levels were decreased, the effect on detection threshold also decreased until there was no longer a detectable effect of $\mathrm{PV}+$ interneuron activation. The small differences in threshold change between animals for a given power of light were expected, due to unavoidable variability in depth, density, and spread of ChR2 expression.

To determine whether the increase in detection threshold was due to a general effect on visual perception or was specific to this contrast detection task, we next directed mice to detect changes in the orientation of a visual stimulus. In this task, a vertically oriented Gabor was continu-

random delay period, a stimulus appears (Gabor patch, $100 \mathrm{~ms}$ duration, $25^{\circ}$ in diameter; eccentricity, $35^{\circ}$ in azimuth, $0^{\circ}$ in elevation; spatial frequency, 0.1 cycle/degree; oriented vertically, odd symmetric), at which point the mouse must release the lever to receive a reward. Stimuli were presented at multiple contrasts to measure the psychophysical detection threshold (Threshold \pm SEM: Mouse A, $10.0 \pm 1.0 \%, n=2$ sessions; Mouse B, $16.9 \pm$ $1.4 \%, n=4$ sessions; Mouse C, $12.0 \pm 0.8 \%, n=9$ sessions; Fig. $1 D)$. These thresholds were $\sim 5-8$ times higher than those previously reported using this same task ( $\sim 2 \%$; Histed et al., 2012). Two factors probably account for this difference. First, we used a much smaller stimulus in the present work to achieve retinotopic specificity $\left(\sim 500 \mathrm{deg}^{2}\right.$ vs $\left.\sim 5000 \mathrm{deg}^{2}\right)$ and contrast sensitivity often scales in proportion to the square root of stimulus area (Rovamo et al., 1994). Second, these smaller stimuli were placed in the monocular visual field, so that only one cortical hemisphere could process the stimuli. Indeed, we observed that thresholds for identical stimuli were lower at central, binocular locations (Threshold \pm SEM, $6.6 \pm 0.5 \%, n=6$ sessions, 2 mice; from data in Fig. $1 F$ ) compared with peripheral, monocular locations (Ipsi, $13.2 \pm 0.7 \%$; Contra, $13.5 \pm 0.9 \%, n=13$ sessions, 2 mice). In any case, the absolute perceptual threshold does not affect our results, as our key observations are threshold comparisons for the same stimulus with and without PV-ChR2 stimulation.

To reveal the contribution of $\mathrm{V} 1$ to this task, on a subset of randomly interleaved trials we excited ChR2-expressing PV + interneurons were excited with blue light $(0.25 \mathrm{~mW})$. When the visual stimulus was retinotopically aligned with cortical suppression, all three mice showed a twofold increase in detection threshold [Threshold increase \pm SEM, $105 \pm 15 \% ; n=3$ mice, 15 sessions; one-sided Komolgorov-Smirnov (KS) test: $p<10^{-6}$; Fig. $1 D, F]$. However, when the visual stimulus was placed either in the center of the screen or ipsilateral to the injection site, we observed no change in detection threshold (center: $1 \pm 5 \%$; $n=$ 2 mice, 6 sessions; one-sided KS test: $p>0.4$; ipsi: $-3 \pm 5 \%$; $n=$ 2 mice, 11 sessions; one-sided KS test: $p>0.9$; Fig. $1 E, F)$. Thus, the behavioral changes were highly specific and occurred only for visual stimuli that had retinotopic positions corresponding to the part of V1 suppressed by activation of PV+ interneurons.

To compare the effect of $\mathrm{PV}+$ interneuron activation across photostimulation levels and across mice, we determined the light power density by measuring the size of the light spot on the dural surface (see Materials and Methods). As light power density increased and thus more inhibition was recruited by ChR2 excitation, visual detection was more strongly impaired, as evidenced by greater elevation of contrast thresholds (Fig. 2A,B). Further, ously displayed, and by varying the stimulus orientation, we found the behavioral threshold for detection of orientation changes (Stimulus contrast $=100 \%$; Orientation change detection threshold \pm SEM: Mouse A, $6.9^{\circ}, n=1$ session; Mouse $\mathrm{B}$, $4.6 \pm 0.1^{\circ}, n=7$ sessions; Mouse C, $5.7 \pm 0.6^{\circ}, n=4$ sessions; Fig. $3 A$ ). In all sessions the three mice had elevated orientation change thresholds when V1 was suppressed by PV + interneuron activation ( $82 \pm 10 \%$ above control; $n=3$ mice, 12 sessions; one-sided KS test: $p<10^{-6}$; Fig. $\left.3 A, D\right)$. In a third behavioral manipulation, we examined whether $V 1$ suppression affected the ability to detect a change in contrast of a medium contrast Gabor (Initial stimulus contrast $=50 \%$ ). We found that activating inhibition also interfered with this task, either when the mouse had to detect an increase (increment threshold elevation \pm SEM: $106 \pm 12 \% ; n=1$ mouse, 3 sessions; one-sided KS test: $p<0.02$ ) or a decrease (decrement threshold elevation \pm SEM: $87 \pm 6 \%$; $n=1$ mouse, 3 sessions; one-sided KS test: $p<0.02$; Fig. $3 B--D$ ) in contrast. Thus, inactivation of $\mathrm{V} 1$ interferes with a variety of detection tasks.

To understand the neural changes accompanying the large behavioral changes we observed, we made extracellular recordings from the site of ChR2 expression in V1. Recorded units had moderate rates of spontaneous activity and responded robustly to the presentation of brief visual stimuli of varying contrast $[n=2$ mice, 2 sessions, 85 of 123 units were visually driven (44 multiunits; 41 single-units; $p<0.001$, Kruskal-Wallis test; see Materials and Methods); Fig. 4A-E]. We then applied light intensities similar to those used in our behavioral task to determine how firing rates were affected. We found that activation of $\mathrm{PV}+$ interneurons profoundly suppressed the spontaneous firing rates of most units (median change in baseline firing rate, $-49.0 \%$; Sign test, $p<10^{-19}$; Fig. $\left.4 F, G\right)$. In comparison, the effect on visually evoked (baseline-subtracted) responses was more modest [median change in threshold contrast (see Materials and Methods), 14.3\%; Sign test, $p<10^{-5}$; Fig. $\left.4 E, G\right]$. We found a weak but significant correlation between the suppression of each unit's spontaneous and evoked firing rate $\left(r^{2}=0.11\right.$, slope $=$ 0.26, $p<0.05$; Fig. $4 G)$.

To determine whether we were suppressing activity in all layers of cortex, we divided our recordings into two groups: superficial ( $\leq 300 \mu \mathrm{m}$ deep; $n=38$ ) and deep ( $>300 \mu \mathrm{m}$ deep; $n=46$ ) units (see Materials and Methods). Recordings at both depths were suppressed by PV + interneuron activation (median change in baseline firing rate, superficial units: $-66.7 \%$; Sign test, $p<$ $10^{-9}$; deep units: $-33.9 \%, p<10^{-9}$; median change in contrast threshold, superficial units: $-24.5 \% ; p<0.001$; deep units: $-11.0 \%, p<0.005)$. The effects were stronger for superficial 
A

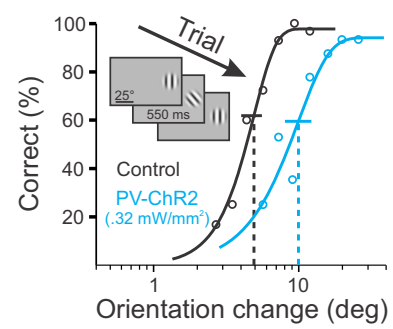

C

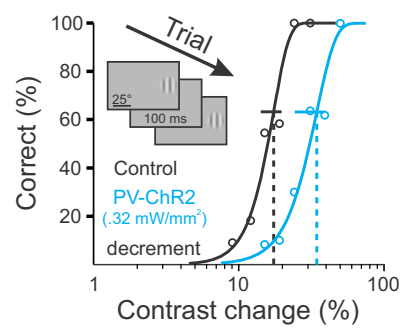

B

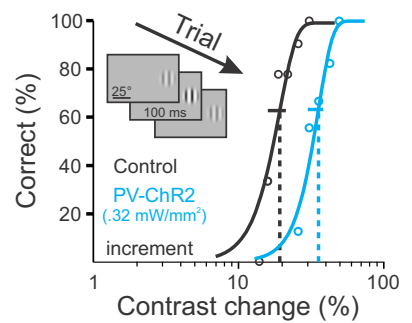

D

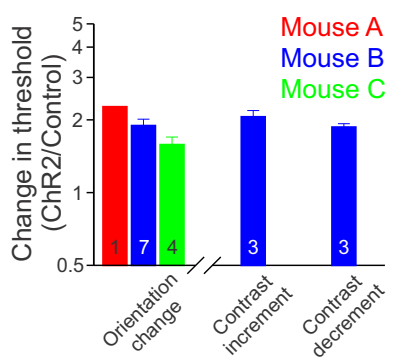

Figure 3. Activation of PV + interneurons degrades performance in multiple detection tasks. $A$, Representative behavioral performance during change in the orientation of a visual stimulus (100\% contrast) in the field of view contralateral to the ChR2 injection site, during control trials (black) and activation of PV + interneurons (blue). Inset, Schematic of visual stimulus. $\boldsymbol{B}$, Same as $\boldsymbol{A}$, for increments in contrast from a baseline contrast of $50 \%$ (same mouse as in $\boldsymbol{A}$ ). C, Same as $\boldsymbol{A}$, for decrements in contrast from a baseline contrast of $50 \%$ (same mouse as in A). D, Summary of effect of activation of $\mathrm{PV}+$ interneurons for the three different tasks (power density: Mouse A, $0.37 \mathrm{~mW} / \mathrm{mm}^{2}$; Mouse B, $0.32 \mathrm{~mW} / \mathrm{mm}^{2}$; Mouse $\left(, 0.46 \mathrm{~mW} / \mathrm{mm}^{2}\right.$ ). Each bar is the average change across the number of behavior sessions indicated. Error bars indicate SEM.

units consistent with scattering and absorption of the blue ChR2activating light as it penetrates the cortex. Thus, the physiological recordings demonstrate that activation of $\mathrm{PV}+$ interneurons has a strongly inhibitory effect on neuronal activity.

\section{Discussion}

Suppression of neuronal activity in mouse V1 increases the threshold for detecting changes in both the orientation and contrast of visual stimuli. This was true even for stimuli with relatively low spatial frequencies $(0.1$ cycle/degree $)$ that were previously thought to be V1-independent (Dean, 1978; Prusky and Douglas, 2004), confirming that mouse V1 is used for computations involved in simple visually guided, operant behaviors. The differences from previous results are likely explained by the difficulty of our near-threshold tasks, which may recruit all available visual neuronal resources, and the reversibility of our cortical suppression, which makes it difficult for animals to adapt and learn to use other brain regions to compensate for $\mathrm{V} 1$ inactivation.

Activation of $\mathrm{PV}+$ interneurons had two distinct effects on the activity of neighboring pyramidal cells: (1) to decrease their baseline firing rates and (2) to decrease their visually evoked responses (Fig. 4). The former alters the peak firing rate while the latter affects the change in firing rate. An ideal observer (Geisler, 2011) would use neuronal response changes relative to the spontaneous level to guide behavior. To do this, other brain areas decoding V1 activity must have knowledge of the spontaneous rate to subtract it from the response. On the other hand, a simpler decoding rule would be a single spike count threshold for detection across both control trials and those with PV + activation. In this case, both spontaneous and response changes could degrade performance. Since we chose to activate the PV + interneurons for the entire window of possible stimulus onsets (to avoid cueing the mice to the stimulus appearance with the ChR2 stimulation), our experiments cannot distinguish whether both effects on neural activity contribute to behavioral changes. However, while we cannot determine the full structure of the decoding strategy used, our physiological data confirm that $\mathrm{PV}+$ interneuron activation on average suppresses neural activity (Fig. 4). Moreover, weak single-neuron effects generated major effects on behavior, suggesting that a large population of neurons contributes to the behavioral task.

Stimulation of PV + interneurons greatly impaired perception (Fig. 1) but even powers that produced maximal effects (1-10 $\mathrm{mW} / \mathrm{mm}^{\wedge} 2$; Fig. $\left.2 B\right)$ could not entirely eliminate visual perception. The remaining visual capability could be due to multiple factors. First, the silenced area may not contain all neurons responsive to the visual stimulus either due to misalignment or the large size and scatter of visual receptive fields (Bonin et al., 2011). Indeed, the ChR2 expression in Mouse A covered $\sim 4$ times more cortical territory than that in Mouse B (1.8 vs 0.5 $\mathrm{mm}^{2}$; likely due to differences in the efficiency of the viral injection), and could therefore explain some of the difference in magnitude of effect at comparable power density. A second possibility is that maximal light activation did not completely suppress V1 activity, either because ChR2 stimulation did not activate PV+ cells sufficiently, or because not all PV + neurons were transfected. Another possibility is that the superior colliculus or another subcortical pathway can support degraded visual detection.

Although changes in the activity of V1 neurons were the root cause of the behavioral change we observed, suppression of activity in V1 undoubtedly causes changes in the activity of V1 targets, such as the superior colliculus, thalamus, and higher visual areas, which may in turn contribute to the behavior. Indeed, lesion or cooling of mammalian V1 has profound effects on visual responses in the colliculus (Wickelgren and Sterling, 1969; Schiller et al., 1974). Nonetheless, our data show that normal activity in $\mathrm{V} 1$ is required for performance of these tasks. Future experiments that silence targets of V1 activity will help to determine the relative contribution of these different structures to detection of visual stimuli.

We never saw improvement in detection threshold with activation of $\mathrm{PV}+$ interneurons, although it is theoretically possible for increased inhibition to improve behavioral performance, for example, by sharpening tuning (for discussion, see Carandini and Ferster, 2000), increasing the number of informative neurons by reducing neuronal gain (Chance et al., 2002), or changing signalto-noise ratio by disproportionately suppressing baseline activity (Fig. $4 F$ ). This is in disagreement with a previous finding that increased inhibition from $\mathrm{PV}+$ interneurons improved orientation discrimination (Lee et al., 2012). However, the data from that other behavioral paradigm are difficult to interpret because those animals show significant improvement even on the comparatively easy trials (i.e., when the stimulus is at $90^{\circ}$ from the target). Because animals are not perceptually limited on easy trials, the fact that they do not achieve near $100 \%$ performance in those cases means that the animals make errors due to cognitive factors like failure to pay attention (Carandini and Churchland, 2013). Thus, it is possible that PV + cell activation could reduce those cognitive errors by increasing arousal or attention. This would improve performance, but might not be directly related to changes in V1 computations. We were able to rule out such global effects of PV + interneuron activation by showing that optogenetic stimulation lost its behavioral effect when the visual and cortical stimulation were misaligned (Fig. 1C). We also trained 
A

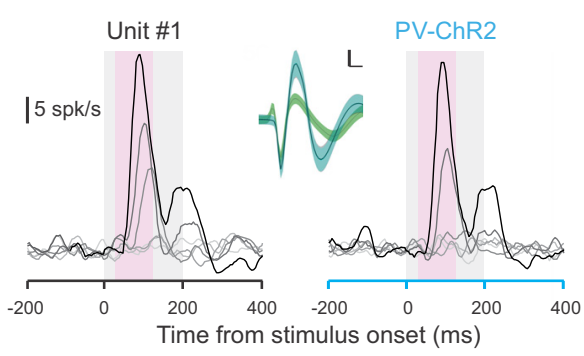

C

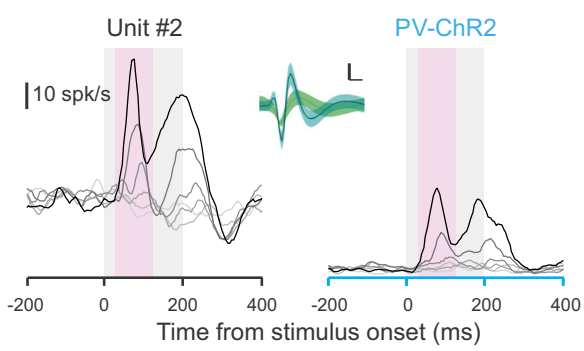

E

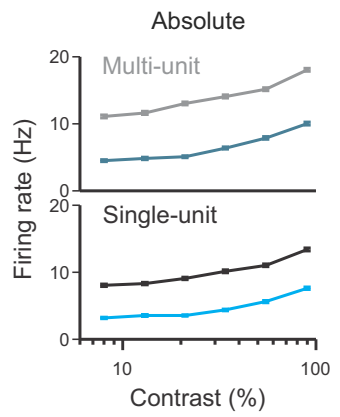

Baseline-subtracted

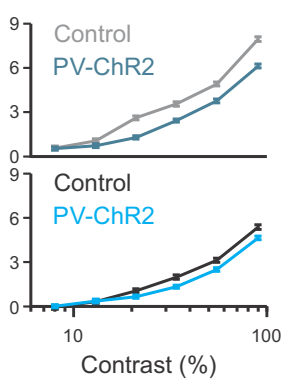

B

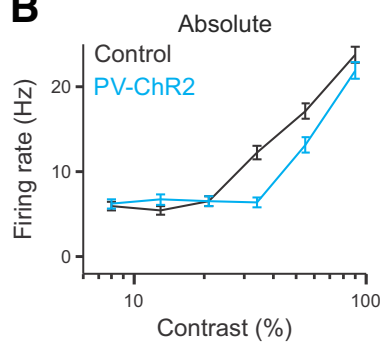

D

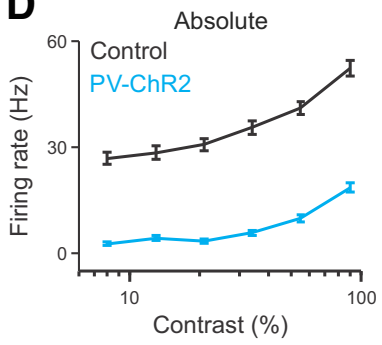

$\mathbf{F}$

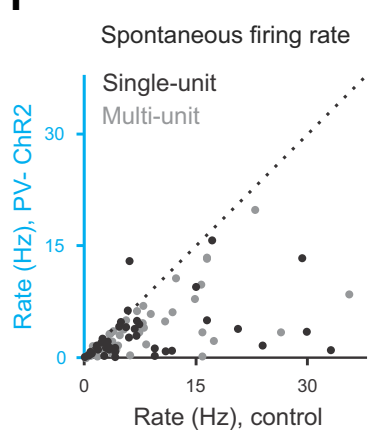

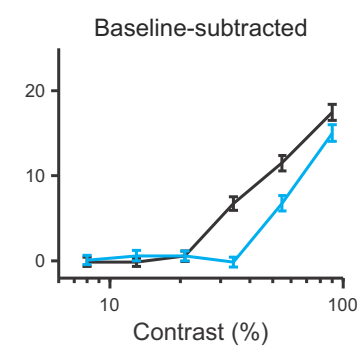

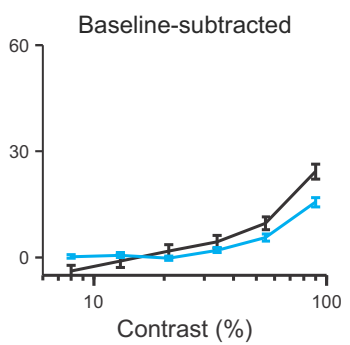

G

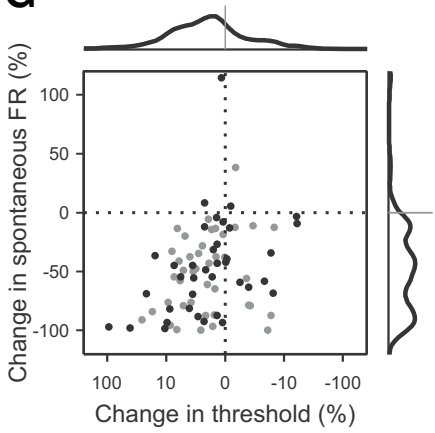

Figure 4. $P V+$ interneuron activation suppresses both baseline firing rate and visual responses. $\boldsymbol{A}, \boldsymbol{B}$, Representative single unit (depth $\sim 400 \mu \mathrm{m}$ ). $\boldsymbol{A}$, Firing rate of unit in response to vertically oriented Gabor patches (200 ms duration, gray shaded epoch) at six contrasts (8, 13, 21, 34, 55, 90\%) for control (left) and ChR2 stimulation (right; 0.62 mW/mm ${ }^{2}$ ) trials. Inset, Average recorded waveform (solid lines) $\pm 1 S D$ (shaded) for spikes recorded from this unit during ChR2 stimulation (blue) or control trials (gray; nearly identical to ChR2 and thus entirely hidden by overlapping blue waveform), and from the next largest unit on this channel (green). Calibration: $50 \mu \mathrm{V}, 0.2 \mathrm{~ms}$. $\boldsymbol{B}$, Average absolute (left) and baseline-subtracted (right) firing rate of unit from $\boldsymbol{A}$ [analysis window (pink shaded epoch in $\boldsymbol{A}$ ): 50-150 ms after stimulus] for control (black) and (hR2 stimulation (blue). $\boldsymbol{C}, \boldsymbol{D}$, Second example single unit (depth $\sim 500 \mu \mathrm{m}$ ). Conventions as in $\boldsymbol{A}$ and $\boldsymbol{B}$. $\boldsymbol{E}$, Population average absolute (left) and visually evoked (right) responses to visual stimuli for multiunits (top, $n=44$ ) and single units (bottom, $n=41$ ) during control (gray/black) and ChR2 stimulation (blue) trials ( $n=2$ mice; $0.6 \mathrm{~mW} / \mathrm{mm}^{2}$ ). Error bars indicate SEM across trials. $F$, Summary of baseline firing rate during control ( $x$-axis) and ChR2 stimulation ( $y$-axis) trials for all single units (black) and multiunits (gray) recorded. Dotted diagonal shows unity. Note that one single unit, a putative ChR2-expressing neuron, increased its firing rate in response to ChR2 stimulation. $\mathbf{G}$, Summary of changes in threshold of visual responses and baseline firing rates with ChR2 stimulation for all units. Histograms (thick lines, top and right) show frequency distributions of each change (both medians significantly less than zero, $p<10^{-5}$, Mann-Whitney test). Two outliers (response change $=+273$ and $-185 \%$ ) were excluded for visual clarity.

animals until they showed high performance on easy stimuli (low lapse rates), suggesting their errors were primarily due to limits of visual perception. While no behavioral measure can completely rule out errors due to inattention, our measured thresholds are reliable day-to-day, giving us confidence that animals are perceptually limited and not affected by fluctuations in motivation. In addition, despite the potential for animals to learn new strategies, these optogenetic lesions had consistent effects on behavior over many months, allowing us to stably assess the role of V1 in behavior. Thus, we can conclude that increased PV + inhibition in visual cortex has a detrimental effect on visual perception.

Activation of $\mathrm{PV}+$ interneurons shifts the psychometric functions such that larger changes in contrast are necessary for the same probability of behavioral response. Thus, the behavioral effect of activation of PV+ interneurons is consistent with a decrease in the apparent contrast of the visual stimulus. Previous studies have also found that effects of activation of PV + interneurons on size tuning were mimicked by decreases in stimulus contrast (Nienborg et al., 2013; Vaiceliunaite et al., 2013). Further, both PV+ interneuron activation (Fig. $3 A, D$ ) and de- creasing the contrast of the stimulus (data not shown) increase the threshold for orientation change detection. This potential effect of PV+ interneuron activation on effective contrast could also explain why the increase in threshold was similar across the different detection tasks (Fig. 3D). Moreover, it suggests a more general perceptual role for PV+ interneurons, and gain control, in determining the effective contrast of visual stimuli.

\section{References}

Andermann ML, Kerlin AM, Roumis DK, Glickfeld LL, Reid RC (2011) Functional specialization of mouse higher visual cortical areas. Neuron 72:1025-1039. CrossRef Medline

Atallah BV, Bruns W, Carandini M, Scanziani M (2012) Parvalbuminexpressing interneurons linearly transform cortical responses to visual stimuli. Neuron 73:159-170. CrossRef Medline

Bonin V, Histed MH, Yurgenson S, Reid RC (2011) Local diversity and fine-scale organization of receptive fields in mouse visual cortex. J Neurosci 31:18506-18521. CrossRef Medline

Carandini M, Churchland AK (2013) Probing perceptual decisions in rodents. Nat Neurosci 16:824-831. CrossRef Medline

Carandini M, Ferster D (2000) Membrane potential and firing rate in cat primary visual cortex. J Neurosci 20:470-484. Medline 
Cardin JA, Carlén M, Meletis K, Knoblich U, Zhang F, Deisseroth K, Tsai LH, Moore CI (2009) Driving fast-spiking cells induces gamma rhythm and controls sensory responses. Nature 459:663-667. CrossRef Medline

Chance FS, Abbott LF, Reyes AD (2002) Gain modulation from background synaptic input. Neuron 35:773-782. CrossRef Medline

Damasio AR, Geschwind N (1984) The neural basis of language. Annu Rev Neurosci 7:127-147. CrossRef Medline

Dean P (1978) Visual acuity in hooded rats: effects of superior collicular or posterior neocortical lesions. Brain Res 156:17-31. CrossRef Medline

Dean P (1981) Grating detection and visual acuity after lesions of striate cortex in hooded rats. Exp brain Res 43:145-153. Medline

Gabernet L, Jadhav SP, Feldman DE, Carandini M, Scanziani M (2005) Somatosensory integration controlled by dynamic thalamocortical feedforward inhibition. Neuron 48:315-327. CrossRef Medline

Geisler WS (2011) Contributions of ideal observer theory to vision research. Vision Res 51:771-781. CrossRef Medline

Haider B, Häusser M, Carandini M (2013) Inhibition dominates sensory responses in the awake cortex. Nature 493:97-100. Medline

Hippenmeyer S, Vrieseling E, Sigrist M, Portmann T, Laengle C, Ladle DR, Arber S (2005) A developmental switch in the response of DRG neurons to ETS transcription factor signaling. PLoS Biol 3:e159. CrossRef Medline

Histed MH, Carvalho LA, Maunsell JHR (2012) Psychophysical measurement of contrast sensitivity in the behaving mouse. J Neurophysiol 107: 758-765. CrossRef Medline

Hofbauer A, Dräger UC (1985) Depth segregation of retinal ganglion cells projecting to the mouse superior colliculus. J Comp Neurol 234:465-474. CrossRef Medline

Kawaguchi Y, Kubota Y (1997) GABAergic cell subtypes and their synaptic connections in rat frontal cortex. Cereb Cortex 7:476-486. CrossRef Medline

Lashley KS (1931a) Mass action in cerebral function. Science 73:245-254. CrossRef Medline

Lashley KS (1931b) The mechanism of vision: IV. The cerebral areas necessary for pattern vision in the rat. J Comp Neurol 53:419-478. CrossRef

Lashley KS (1939) The mechanism of vision: XVI. The functioning of small remnants of the visual cortex. J Comp Neurol 70:45-67. CrossRef

Lee SH, Kwan AC, Zhang S, Phoumthipphavong V, Flannery JG, Masmanidis SC, Taniguchi H, Huang ZJ, Zhang F, Boyden ES, Deisseroth K, Dan Y (2012) Activation of specific interneurons improves V1 feature selectivity and visual perception. Nature 488:379-383. CrossRef Medline

Nagel G, Szellas T, Huhn W, Kateriya S, Adeishvili N, Berthold P, Ollig D, Hegemann P, Bamberg E (2003) Channelrhodopsin-2, a directly lightgated cation-selective membrane channel. Proc Natl Acad Sci U S A 100: 13940-13945. CrossRef Medline

Nienborg H, Hasenstaub A, Nauhaus I, Taniguchi H, Huang ZJ, Callaway EM
(2013) Contrast dependence and differential contributions from somatostatin- and parvalbumin-expressing neurons to spatial integration in mouse V1. J Neurosci 33:11145-11154. CrossRef Medline

O'Connor DH, Hires SA, Guo ZV, Li N, Yu J, Sun QQ, Huber D, Svoboda K (2013) Neural coding during active somatosensation revealed using illusory touch. Nat Neurosci 16:958-965. CrossRef Medline

Packer AM, Yuste R (2011) Dense, unspecific connectivity of neocortical parvalbumin-positive interneurons: a canonical microcircuit for inhibition? J Neurosci 31:13260-13271. CrossRef Medline

Petruno SK, Clark RE, Reinagel P (2013) Evidence that primary visual cortex is required for image, orientation, and motion discrimination by rats. PLoS One 8:e56543. CrossRef Medline

Prusky GT, Douglas RM (2004) Characterization of mouse cortical spatial vision. Vision Res 44:3411-3418. CrossRef Medline

Rovamo J, Mustonen J, Risto N, Nen S (1994) Modelling contrast sensitivity as a function of retinal illuminance and grating area. Vision Res 34:1301-1314.

Schiller PH, Stryker M, Cynader M, Berman N (1974) Response characteristics of single cells in the monkey superior colliculus following ablation or cooling of visual cortex. J Neurophysiol 37:181-194. Medline

Schiller PH, True SD, Conway JL (1980) Deficits in eye movements following frontal eye-field and superior colliculus ablations. J Neurophysiol 44:1175-1189. Medline

Schneider GE (1969) Two visual systems. Science 163:895-902. CrossRef Medline

Sohal VS, Zhang F, Yizhar O, Deisseroth K (2009) Parvalbumin neurons and gamma rhythms enhance cortical circuit performance. Nature 459: 698-702. CrossRef Medline

Tanahira C, Higo S, Watanabe K, Tomioka R, Ebihara S, Kaneko T, Tamamaki N (2009) Parvalbumin neurons in the forebrain as revealed by parvalbumin-Cre transgenic mice. Neurosci Res 63:213-223. CrossRef Medline

Urban A, Rancillac A, Martinez L, Rossier J (2012) Deciphering the neuronal circuitry controlling local blood flow in the cerebral cortex with optogenetics in PV::Cre transgenic mice. Front Pharmacol 3:105. CrossRef Medline

Vaiceliunaite A, Erisken S, Franzen F, Katzner S, Busse L (2013) Spatial integration in mouse primary visual cortex. J Neurophysiol 110:964-972. CrossRef Medline

Wickelgren BG, Sterling P (1969) Effect on the superior colliculus of cortical removal in visually deprived cats. Nature 224:1032-1033. CrossRef Medline

Wilson NR, Runyan CA, Wang FL, Sur M (2012) Division and subtraction by distinct cortical inhibitory networks in vivo. Nature 488:343-348. CrossRef Medline 